\title{
Analysis of Differences in User Behavior Based on a Variety of Online Social Networks
}

\author{
Fenglian Jiang, ${ }^{*}$ Wencan Tong, and Liming Huang \\ School of Mathematics and Information Engineering, Longyan University, Longyan City, China
}

(Received November 23, 2018; accepted February 19, 2019)

Keywords: social network, user behavior, mass data

The development of the Internet and various communication technologies has led to novel innovations, with a variety of social networks and smart terminals prevailing, which has created a vast amount of real data for analyzing and predicting user behaviors. Here, we will analyze and compare multiple online social networks and related user behaviors, giving an introduction to important research from related fields. We will create a model for the analysis of differences in user behavior based on a comparison of multiple online social networks. Differences in user behavior will also be shown on the basis of this model.

\section{Introduction}

The term "online social networks" refers to social service networks that are based on building interpersonal relationships and are propagated over the Internet. Facebook, which went online in 2004 and offers online social services based on real interpersonal relationships, is considered to be the first online social network. With Facebook as the representative, social networks have rapidly developed, and an increasing number of social network platforms are emerging. At the same time, the number of people joining social networks is growing. By 2012, Facebook had more than 1 billion registered users and Sina Weibo had more than 0.3 billion users, which means that social networks have entered people's everyday lives.

Nowadays, the most popular social networks include Facebook, Twitter, Line, QQ, Weibo, and Zhihu. After several years of development, these platforms all offer various functions while essentially progressing toward comprehensive platforms. However, in reality, each platform has its own limitations in terms of service functions, regardless of the perspectives of the users or service providers. This means that for a typical user, it is likely that he or she will register with several social networks at the same time to meet different requirements in different situations.

There is much research value in the coexistence of these various platforms. For example, recent studies on the behaviors of social network users and information propagation have focused on Facebook and Weibo, with comparisons of social networks having different media and vertical characteristics being neglected. Furthermore, analyzing the differences in user behavior in various social networks such as Weibo, Tieba, Zhihu, and Wechat is expected to be a future hot topic.

*Corresponding author: e-mail: jf16868@126.com https://doi.org/10.18494/SAM.2019.2222 
In the rest of this paper, we will compare various social networks and analyze differences in user behavior between these different social networks. Recent important research in related fields will be introduced in Sect. 2. Various online social networks will be compared in Sect. 3. A model for analyzing differences in user behavior in various social networks will be presented in Sect. 4. A simulation experiment will be discussed in Sect. 5, and the paper will be concluded in Sect. 6.

\section{Related Research}

Mobile social networks have grown from nonexistent to ubiquitous in just over a decade. With this rapid development, many academics have become interested in research on social networks. The studies on social networks, both domestic and abroad, which have been carried out to date, can be divided into several aspects: personalized searches, precision marketing, friend recommendations, user behavior analysis, and research on influence and social graphs. ${ }^{(1-4)}$

Precision marketing: Advertisements are the main source of revenue for Internet companies, with a large share of Internet companies' profits based on ads. Thus far, these ads have been randomly inserted into various media with very low efficiency. This has created a lot of opportunities for companies to research the targeting of ads, and this is what is meant by precision marketing.

Friend recommendations: Friendship is a crucial part of social networks. If a site has a poor friendship recommendation function, the user experience is likely to be unsatisfactory, which means that friend recommendation is an important application for social network sites. To date, considerable progress has been achieved on friend recommendation algorithms in social networks, such as friend recommendations based on association rules and those based on collaborative filtering.

User behavior analysis: According to recent research, user behaviors are not random but based on many designated models. For example, when we browse e-commerce sites, there must be a shopping cart provided for customers to store what they will buy, which means that shopping cart analysis is the core data analysis business model for many e-commerce sites. Kinds of example are persuasive if given, and user behavior analysis aims to identify certain user habits through data mining algorithms. ${ }^{(3,6)}$

Research on influence and social graphs: Influence may have various forms of proposal or dominance, which depends on the structural type of the social network and shows different forms and strengths. Scholars have applied algorithms from other fields to study influence in social networks. For example, Facebook has put resources into social graphs and social network structures, while Sina Weibo's main social network characteristic is hobby graphs, and studies by Sina Weibo include the development of a social network user hobby model.

A substantial number of algorithms are based on the analysis of user behavior in social networks, and preference predictions are based on predicting the preference relationships of interusers. First, they calculate preference similarity based on intersocial users, then pick users that most closely approximate the targeted users, called "nearest-neighbor users". They then use the behavior preferences of these nearest-neighbor users as the benchmarks for targeted 
predictors of user behavior, with different approximation strengths weighed as the behavior preference trends of the targeted user. Thus far, there have been many computations on similarity, including Pearson's correlation coefficient and the mean squared error. ${ }^{(1-4)}$ Social networks can be substantially described as graphs, so prediction algorithms based on a graph model are presented here. ${ }^{(5-8)}$

\section{Comparison of Online Social Networks}

Online social network platforms have matured after being developed for over a decade, with each platform becoming more professional and comprehensive. Each popular social network platform has its own irreplaceable business model allowing platforms to coexist with each other, although they may have services overlapping with other platforms. We need to list the characteristics of each online social network platform to make a comparison, with an eye toward the differences between these characteristics.

\subsection{Characteristics of online social networks}

Here, we introduce various functions and meanings of social network platforms.

Social: Social refers to interpersonal services, and the fundamental role of each social network is to build human relationships. Although every social network provides basic human relationship creation services, each platform has its own focus.

For online social platforms such as Facebook and Renren, social services are the most important. These platforms are motivated to encourage real-name social activities, with many social functions such as adding common friends and those with a common focus.

However, information-publishing platforms such as Weibo and Twitter pay more attention to media functions. Although every social network has improved its social services over time, when users want to enjoy intimate social activities with their friends, they have their own personal preferences. ${ }^{(9,10)}$

Medium: Medium refers to the characteristics that social networks have to make and publish valuable content. The creation of social networks has given many opportunities to network providers, such as media for publishing platforms and new media technology. Social networks serve as important avenues for individuals and organizations to publish information, share their opinions, and carry out marketing, with their medium characteristics increasingly being more focused on.

Platforms such as Sina Weibo and Twitter are social networks with strong medium characteristics. Compared with communications between private individuals, opinion leaders in social networks have a widespread influence on information propagation among netizens, and may even accidentally use their influence in reality. The salt snapping event in 2011 is one such negative result of social networks with medium characteristics.

Instancy: With online social networks serving as platforms for deepening human relationships, their basic services allow individual communication. In the case of instant apps such as QQ, Line, and Wechat, user communication is the foundation of their models. 
Instancy refers to the ability of platforms to provide real-time communications. Although every social network provides some kind of real-time communication service, QQ, Wechat, and Line are the mainstream players in the market owing to their high quality and massive user bases.

Community: A community provides multi-to-multi services, where users having common interests can form groups to communicate about certain topics, which have become important parts of social networks. This is in complete contrast to individual-to-individual communication services or blog services.

Community characteristics refer to the multi-to-multi communication services that social networks can provide. Forums and groups are representatives of community services. They have the characteristics of user stickiness while improving group communications.

Multimedia character: Videos and images have long been inseparable parts of social networks, and the multimedia characteristic refers to the ability of social networks to provide multimedia services.

Although each social platform tries to enrich its products via multimedia services, social networks aimed at single types of media have also become popular, such as the image social networks Instagram and Flickr, the music social network Last.fm, and the video social network YouTube, which all have more than 0.1 billion users.

Verticality: Verticality refers to the characteristics of profession and convenience, which are aimed at satisfying the particular requirement of minorities. Social networks with verticality characteristics can provide professional and personal services, in which they try to do business in segmented fields.

Some well-known social networks with verticality characteristics include Tiexue, which offers military information, MOMO, which aims to provide social service between strangers, and social networks focused on knowledge such as Quora, Zhihu, and Douban. They all achieve designated crowd support for their professional and personal services.

\subsection{Well-known social networks used for comparison}

After listing the six characteristics of representative online social networks, we can observe the differences between these social networks through comparisons based on these six dimensions. In Table 1, we will attempt to compare these social networks on the basis of Business Insider's global 10 social network rankings.

We can easily find differences between social networks through comparisons of these six dimensions. Next, we will discuss the relationship between social network and user behavior differences.

\section{Model Used for Analysis of Differences in User Behaviors in Online Social Networks}

The infrastructure of social network deployment is based on relationships among users, and the active users who lead interactive behaviors in social networks are the core part of each social 
Table 1

Popular social networks for comparisons.

\begin{tabular}{lcccccc}
\hline Site & Social & Medium & Instancy & Community & Multimedia & Verticality \\
\hline Facebook & Strong & Strong & Medium & Strong & Middle & Weak \\
YouTube & Weak & Strong & Weak & Weak & Strong & Strong \\
QQ Zone & Strong & Middle & Strong & Middle & Middle & Weak \\
Sina Weibo & Middle & Strong & Middle & Weak & Middle & Weak \\
WhatsApp & Weak & Weak & Strong & Weak & Weak & Weak \\
Google+ & Middle & Middle & Middle & Strong & Middle & Middle \\
Tumblr & Middle & Middle & Middle & Weak & Middle & Middle \\
Line & Middle & Weak & Strong & Middle & Weak & Weak \\
Twitter & Middle & Strong & Middle & Weak & Middle & Weak \\
Wechat & Strong & Middle & Strong & Middle & Middle & Weak
\end{tabular}

network. Analyzing user behaviors will lead to a better understanding of social networks, help improve social network platform interface designs, and aid research on the mutual activities of societies.

To study user behaviors, we need to focus on users in a variety of social network platforms. As every platform provides different services, we need a united standard of user behavior to design a benchmark based on the basic interactive behaviors of users. Thus, we need to discuss what behaviors users exhibit before creating the model.

\subsection{Common user behaviors}

Online social network user behaviors can be divided into two categories, explicit and implicit behaviors, and the differences between them should be analyzed if explicit interactive data exist. Explicit behaviors include messages and comments that can be seen by visitors, while implicit behaviors do not leave any explicit messages, with visiting homepages being a typical example. Both can be sensed through website mouse click stream data. Thus, here, we define user behaviors as at least one HTTP request activity in mouse click stream data, with Facebook as an example, and the 40 common online social network user behaviors listed in Table 2. ${ }^{(1)}$

User manipulations on online social network platforms through mouse clicks can be summarized into the 40 behaviors in Table 2. Although the exact names or concepts for user behaviors may differ across the various platforms, all the behaviors can be found in Table 2 . To reduce the variation so as to facilitate model creation while setting a standard for various platforms, these 40 items will be integrated.

\subsection{Integration of user behaviors}

We divide user behaviors into nine categories based on object manipulation: Search, Post, Private Message, Message, Video, Image, Management, Community, and Configuration. Each can be defined as follows: 
Table 2

List of user behaviors.

\begin{tabular}{|c|c|c|c|}
\hline User behavior & Description & User behavior & Description \\
\hline General search & $\begin{array}{l}\text { Information search by } \\
\text { keywords }\end{array}$ & $\begin{array}{l}\text { Browse a friend } \\
\text { update }\end{array}$ & $\begin{array}{l}\text { Browse friend's } \\
\text { update moments }\end{array}$ \\
\hline Visit a post & $\begin{array}{l}\text { Browse own or } \\
\text { friend's post }\end{array}$ & $\begin{array}{l}\text { Browse community } \\
\text { members }\end{array}$ & $\begin{array}{l}\text { Browse members of } \\
\text { friend's community }\end{array}$ \\
\hline Edit a post & Edit a new post & Edit a moment & Edit own moment \\
\hline $\begin{array}{l}\text { Browse a private } \\
\text { message }\end{array}$ & $\begin{array}{l}\text { Browse messages } \\
\text { received }\end{array}$ & Browse a user list & $\begin{array}{l}\text { Browse a list of } \\
\text { strangers }\end{array}$ \\
\hline $\begin{array}{l}\text { Edit a private } \\
\text { message }\end{array}$ & Edit private messages & User management & $\begin{array}{l}\text { Management of users } \\
\text { except friends }\end{array}$ \\
\hline $\begin{array}{l}\text { Browse } \\
\text { comments }\end{array}$ & $\begin{array}{l}\text { Browse comments } \\
\text { left by friends }\end{array}$ & $\begin{array}{l}\text { Browse a } \\
\text { community }\end{array}$ & Browse a community \\
\hline Edit a message & $\begin{array}{l}\text { Edit a message } \\
\text { for friends }\end{array}$ & $\begin{array}{l}\text { Browse community } \\
\text { comments }\end{array}$ & $\begin{array}{l}\text { Browse community } \\
\text { comments }\end{array}$ \\
\hline $\begin{array}{l}\text { Browse a past } \\
\text { message }\end{array}$ & $\begin{array}{l}\text { Browse messages } \\
\text { read }\end{array}$ & $\begin{array}{l}\text { Join/leave a } \\
\text { community }\end{array}$ & $\begin{array}{l}\text { Join or leave a } \\
\text { community }\end{array}$ \\
\hline Browse video list & $\begin{array}{l}\text { Browse a } \\
\text { recommended video list }\end{array}$ & $\begin{array}{l}\text { Browse community } \\
\text { members }\end{array}$ & $\begin{array}{l}\text { Browse a community } \\
\text { members }\end{array}$ \\
\hline Browse a video & Browse a video of interest & Browse a topic list & $\begin{array}{l}\text { Browse community } \\
\text { topic list }\end{array}$ \\
\hline $\begin{array}{l}\text { Browse a photo } \\
\text { album list }\end{array}$ & $\begin{array}{l}\text { Browse an available } \\
\text { photo album list }\end{array}$ & Join in a topic discussion & $\begin{array}{l}\text { Post in a community } \\
\text { topic }\end{array}$ \\
\hline $\begin{array}{l}\text { Browse a photo } \\
\text { album }\end{array}$ & Browse a photo album & Community management & $\begin{array}{l}\text { Community } \\
\text { management }\end{array}$ \\
\hline $\begin{array}{l}\text { Browse an } \\
\text { image }\end{array}$ & Browse an image & Vote & Vote for a community \\
\hline $\begin{array}{l}\text { Browse tagged } \\
\text { images }\end{array}$ & $\begin{array}{l}\text { Browse tagged images } \\
\text { annotated by self or friends }\end{array}$ & $\begin{array}{l}\text { Browse a community } \\
\text { list }\end{array}$ & $\begin{array}{l}\text { Browse communities } \\
\text { joined }\end{array}$ \\
\hline $\begin{array}{l}\text { Browse image } \\
\text { comments }\end{array}$ & Browse image comments & $\begin{array}{l}\text { Community management } \\
\text { invitation }\end{array}$ & $\begin{array}{l}\text { Apply for or accept a } \\
\text { community invitation }\end{array}$ \\
\hline Edit a photo & Edit a published photo & Community activities & $\begin{array}{l}\text { Join in a community } \\
\text { activity }\end{array}$ \\
\hline Browse states & $\begin{array}{l}\text { Browse own or } \\
\text { friend's states }\end{array}$ & Visit applications & $\begin{array}{l}\text { Visit an application } \\
\text { the platform offers }\end{array}$ \\
\hline $\begin{array}{l}\text { Browse a } \\
\text { homepage }\end{array}$ & $\begin{array}{l}\text { Browse own or } \\
\text { friend's homepage }\end{array}$ & User configuration & User configuration \\
\hline $\begin{array}{l}\text { Browse friend } \\
\text { list }\end{array}$ & Browse own friend list & Spam/verification code & Browse spam \\
\hline $\begin{array}{l}\text { Manage friend } \\
\text { invitation }\end{array}$ & Accept a friend request & User log in or log out & User log in or log out \\
\hline
\end{tabular}

Search: Search refers to a general search, for providing a post, moment search, community, topic search. Users can find what they need by searching, for example, targeted users, communities, posts, images, and videos.

Post: A post has a different name on each social platform. It is called a post in Facebook, a Weibo in Sina Weibo, a Tweet in Twitter, and a Shuoshuo in QQ. In general, a post is publicly published by users and can be seen by anybody (who has reading authorization), in contrast to a private message or email. Posting is the basic way for users to show who they are and to express themselves; of course, it has the most important position on social platforms. 
Private Message: Private messaging refers to the exchange of private information between individuals. Private messages dominate instant message social networks, such as QQ, Wechat, and Line, and have auxiliary functions in forums, which are focused on communities.

Message: Messaging includes leaving a message for friends and responding to friends' comments, and it is a major component of public behavior. Messages are created by users and sent to each other, but are open to others, although the owners can also set viewing authorizations.

Video and Image: Videos and images include all multimedia contents. Similarly to text, multimedia are open to everyone, can be tagged by others, and authorizations can be set.

Management: Management includes user management, friend management, and friend visit management. Unlike posts, private messages, and messages, which all have their own publishing objects, moments have no publishing objects, but provide other user information, such as user homepages, signatures, profiles, visit logs, and geographical positions. Moments can be public to others on the basis of authorizations. User management includes friend applications, requests, and deletions while editing and visiting.

Community: Communities play an important role in social networks. Registered members of a community can set a topic, exchange information with each other, set a vote or question, or establish games within communities. Common communities include forums and QQ/Line/ Wechat groups.

Configuration: User configuration refers to all behaviors related to the configuration by users on social networks, including the use of applications, spam shielding and deletion, and logging in/out

We can create a model to analyze the differences between user behaviors on multiple social network platforms on the basis of the nine characteristics mentioned above, with user behaviors compared across the different social network platforms.

\subsection{Model used to analyze differences in user behavior}

We can create a model to analyze differences in user behavior through the multivariate analysis of variance (MANOVA) using the categories listed above.

Imagine we have mouse click stream data sets from social networks $\mathrm{A}, \mathrm{B}, \mathrm{C}$, and $\mathrm{D}$, and randomly pick 200 users from each set. The user behavior parameters are shown in Table 3. One month parameter data are shown in Table 4. Different times parameter coefficients are shown in Table 5. Then, we calculate the cumulative counts of eachbehavior across one week.

First, we calculate the total variance as follows before creating a model of difference analysis by MANOVA:

$$
\sum_{i} \sum_{j}\left(Y_{i j}-G M\right)^{2}=n \sum_{j}\left(\bar{Y}_{j}-G M\right)^{2}+\sum_{i} \sum_{j}\left(Y_{i j}-\mid \overline{Y_{j}}\right)^{2}
$$


Table 3

User behavior parameters.

\begin{tabular}{cccccccccc}
\hline & Search & Text & Message & Testimonial & Videos & Photos & Manage & Communities & Setting \\
\hline a & A1 & A2 & A3 & A4 & A5 & A6 & A7 & A8 & A9 \\
b & B1 & B2 & B3 & B4 & B5 & B6 & B7 & B8 & B9 \\
c & C1 & C2 & C3 & C4 & C5 & C6 & C7 & C8 & C9 \\
d & D1 & D2 & D3 & D4 & D5 & D6 & D7 & D8 & D9 \\
\hline
\end{tabular}

Table 4

User behavior parameter data (one month).

\begin{tabular}{cccccccccccc}
\hline & Search & Text & Message & Testimonial & Videos & Photos & Manage Communities & Setting & Average & Total variance \\
\hline a & 59 & 38 & 89 & 40 & 55 & 67 & 43 & 98 & 34 & 58.11 & 21.54 \\
b & 88 & 45 & 90 & 60 & 45 & 78 & 65 & 76 & 25 & 63.56 & 20.68 \\
c & 109 & 56 & 130 & 78 & 67 & 98 & 76 & 98 & 14 & 80.67 & 31.81 \\
d & 78 & 28 & 38 & 45 & 38 & 65 & 23 & 34 & 24 & 41.44 & 17.66 \\
\hline
\end{tabular}

Table 5

User behavior parameter coefficients (different times).

\begin{tabular}{lcccccccc}
\hline & $\begin{array}{c}\text { Average } \\
(30 \mathrm{~d})\end{array}$ & $\begin{array}{c}\text { Average } \\
(60 \mathrm{~d})\end{array}$ & $\begin{array}{c}\text { Average } \\
(90 \mathrm{~d})\end{array}$ & $\begin{array}{c}\text { Average } \\
(120 \mathrm{~d})\end{array}$ & $\begin{array}{c}\text { Total variance } \\
(30 \mathrm{~d})\end{array}$ & $(60 \mathrm{~d})$ & $(90 \mathrm{~d})$ & $(120 \mathrm{~d})$ \\
\hline $\mathrm{a}$ & 58.11 & 60.12 & 62.51 & 60.52 & 21.54 & 22.82 & 22.02 & 22.85 \\
$\mathrm{~b}$ & 63.56 & 70.35 & 72.51 & 75.12 & 20.68 & 23.41 & 22.64 & 23.01 \\
$\mathrm{c}$ & 80.67 & 75.62 & 86.71 & 85.12 & 31.81 & 30.21 & 30.55 & 30.12 \\
$\mathrm{~d}$ & 41.44 & 35.42 & 38.66 & 40.12 & 17.66 & 18.12 & 17.52 & 18.52 \\
\hline
\end{tabular}

Then, we calculate the interclass variance:

$$
\begin{aligned}
& n_{k m} \sum_{k} \sum_{m}\left(D T_{k m}-G M\right)^{2}=n_{k} \sum_{k}\left(D_{k}-G M\right)^{2}+n_{m} \sum_{m}\left(T_{m}-G M\right)^{2} \\
& +\left[n_{k m} \sum_{k} \sum_{m}\left(D T_{k m}-G M\right)^{2}-n_{k} \sum_{k}\left(D_{k}-G M\right)^{2}-n_{m} \sum_{m}\left(T_{m}-G M\right)^{2}\right] .
\end{aligned}
$$

We replace $D T$ with function $Y$ to calculate

$$
\begin{aligned}
& \sum_{i} \sum_{k} \sum_{m}\left(Y_{i k m}-G M\right)^{2}=n_{k} \sum_{k}\left(D_{k}-G M\right)^{2}+n_{m} \sum_{m}\left(T_{m}-G M\right)^{2} \\
& +\left[n_{k m} \sum_{k} \sum_{m}\left(D T_{k m}-G M\right)^{2}-n_{k} \sum_{k}\left(D_{k}-G M\right)^{2}-n_{m} \sum_{m}\left(T_{m}-G M\right)^{2}\right] \\
& +\sum_{i} \sum_{k} \sum_{m}\left(Y_{i k m}-D T_{k m}\right)^{2} .
\end{aligned}
$$


Then, we calculate the significance coefficient lambda $(\wedge)$ as

$$
\wedge=\frac{\left|S_{\text {error }}\right|}{\left|S_{\text {effect }}+S_{\text {error }}\right|} .
$$

Finally, we list the significantly different coefficients of the model in Table 6 . We can find the specific difference variances of each website from these coefficients.

\section{Simulation Experiment and Results}

Assume that A, B, C, and D are behavior data created on social platforms over one month. The changes in the behavior of user A with time are shown in Fig. 1. We set the parameters as shown in Fig. 2 on the basis of fundamental data to analyze user differences.

In social networks, differences in user behavior habit also cause different behavior characteristics. In Figs. 2 and 3, the behaviors of different users are shown to vary within a certain range.

Table 6

Significantly different coefficients of the model.

\begin{tabular}{lcccc}
\hline Source of variance & $\begin{array}{c}\text { Wilks' } \\
\text { Lambda }\end{array}$ & $d f_{1}$ & $d f_{2}$ & $\begin{array}{c}\text { Multivariate } \\
F\end{array}$ \\
\hline Treatment & 0.13772 & 2.00 & 11.00 & $34.43570^{* *}$ \\
Disability & 0.25526 & 4.00 & 22.00 & $5.38602^{*}$ \\
Treatment by disability & 0.90807 & 4.00 & 22.00 & 0.2717 \\
\hline
\end{tabular}

${ }^{*} p<.01 .{ }^{* *} p<.001$.

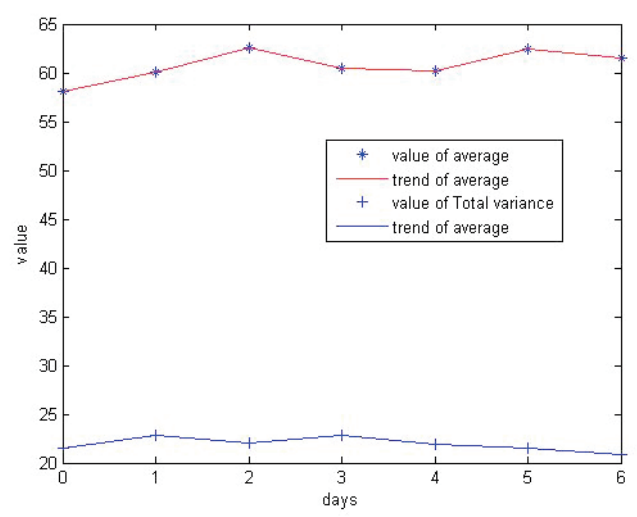

Fig. 1. (Color online) Curve showing behavior changes in behavior of user $\mathrm{A}$.

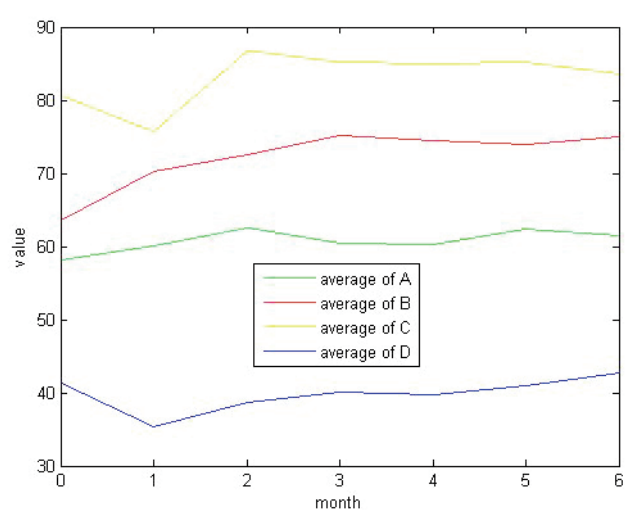

Fig. 2. (Color online) Change in mean behavior of different users over time. 


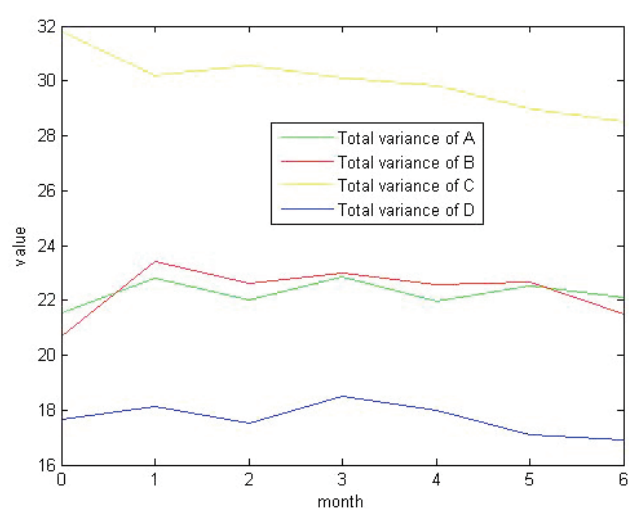

Fig. 3. (Color online) Total variance of the behavior characteristic for different users over time.

\section{Conclusions}

By creating a model to compare user behaviors in social networks, in this paper, we reveal that there are relationships among user behavior characteristics based on social networks, deployments are based on relationships among users, and the active users who lead interactive behaviors in social networks are the cores of social networks. Analyzing user behaviors is an effective means of increasing knowledge on social networks, improving the user interface designs of social networks, and assisting research on social interactivity. The results of this study may be meaningful for future research on social-network-related models.

\section{Acknowledgments}

This work was supported by the Science and Technology Project of Longyan City (2017LY74), Longyan University (LQ2016004), and the Youth Project of Fujian Province Education Hall (JT180522).

\section{References}

1 J. S. Breese, D. Heckerman, and C. Kadie: Proc. 14th Annu. Conf. Uncertainty in Artificial Intelligence 7 (2013) 43.

2 C. Fidel and V. Formoso: ACM Trans. Web 5 (2011) 1.

3 Koutrika, Georgia, B. Bercovitz, and H. Garcia-Molina: Proc. ACM SIGMOD Int. Conf. Management of Data (ACM, 2009) 745.

4 Herlocker and L. Jonathan: SIGIR'99: Proc. Int. ACM SIGIR Conf. Research and Development in Information Retrieval, DBLP (1999) 230.

5 J.-H. Kang, K. Lerman, and L. Getoor: Proc. 6th Int. Conf. Social Computing, Behavioral-Cultural Modeling, and Prediction (AAAI, 2013) 211.

6 K. Lerman: IEEE Internet Comput. 11 (2007) 16.

7 S. Doerfel, D. Zoller, P. Singer, T. Niebler, A. Hotho, and M. Strohmaier: ACM Trans. Web 10.2 (2016) 14.

8 A. E. van Asselt Goverts: J. Autism Dev. Disord. 45 (2015) 1.

9 T. Hogg, K. Lerman, and L. M. Smith: 2013 Int. Conf. Social Computing (IEEE, 2013) 63.

10 K. D. Locke: Pers. Soc. Psychol. Bull. 31 (2005) 79.

11 F. Benevenuto, T. Rodrigues, M. Cha, and V. Almeida: Proc. 9th ACM SIGCOMM Conf. Internet Measurement (ACM, 2009) 49. 


\section{About the Authors}

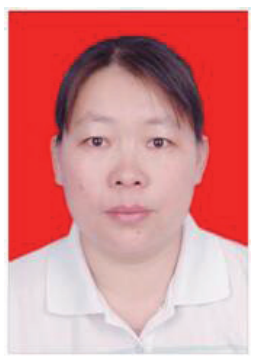

Feng-Lian Jiang received her B.S. degree in Computer Science from Fujian Normal University in 1992 and her M.S. degree in Computer Science in 2009. She teaches in the School of Mathematics and Information Engineering of Longyan University, which is based in Longyan, Fujian, China. She now serves as an associate professor and the head of the Computer Science Department. Her main research interests include computer science, data application, and network information security.

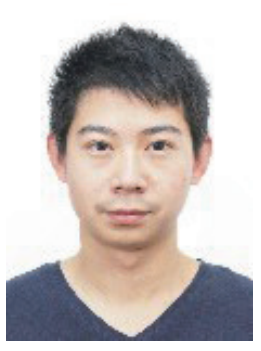

Wen-Can Tong received his B.S. degree in Computer Science and Technology from Huaqiao University in 2011 and his M.S. degree in Computer Application Technology in 2015. In Aug 2016, he served as a lecturer and the deputy director of the Internet of Things Department of the School of Mathematics and Information Engineering of Longyan University, which is based in Longyan, Fujian, China. His main research interests include computer science, network and information security, internet of things, and WSN.

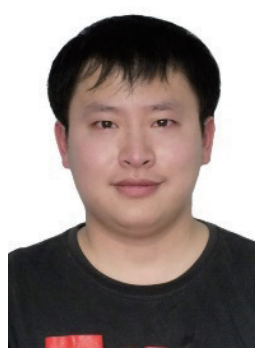

Li-Ming Huang received his B.S. degree in Software Engineering from Jiangxi Normal University in 2011 and his M.S. degree in Computer Technology from Xiamen University in 2014. He joined the School of Mathematics and Information Engineering of Longyan University, which is based in Longyan, Fujian, China, in Aug 2015 and now serves as a lecturer. His main research interests include software architecture, machine learning, and computer graphics. 